\title{
TRACE FUNCTIONS OF THE PARAFERMION VERTEX OPERATOR ALGEBRAS
}

\author{
CHONGYING DONG, VICTOR KAC, AND LI REN
}

\begin{abstract}
The trace functions for the Parafermion vertex operator algebra associated to any finite dimensional simple Lie algebra $\mathfrak{g}$ and any positive integer $k$ are studied and an explicit modular transformation formula of the trace functions is obtained.
\end{abstract}

\section{INTRODUCTION}

This paper is a continuation of our study of the Parafermion vertex operator algebra $K(\mathfrak{g}, k)$ associated to any finite dimensional simple Lie algebra $\mathfrak{g}$ and positive integer $k$. In particular, we determine the modular transformation formula of the trace functions for the Parafermion vertex operator algebras.

The Parafermion vertex operator algebra $K(\mathfrak{g}, k)$ is the commutant of the Heisenberg vertex operator algebra $M_{\widehat{\mathfrak{h}}}(k)$ in the affine vertex operator algebra $L_{\mathfrak{g}}(k, 0)$, and can also be regarded as the commutant of the lattice vertex operator algebra $V_{\sqrt{k} Q_{L}}$ in $L_{\widehat{\mathfrak{g}}}(k, 0)$ where $Q_{L}$ is the lattice spanned by the long roots of $\mathfrak{g}$. While the $C_{2}$-cofiniteness of the Parafermion vertex operator algebras was proved in [20] and [2], the rationality was established in [17] with the help of a result in [5] on the abelian orbifolds for rational and $C_{2}$-cofinite vertex operator algebras. The irreducible modules of $K(\mathfrak{g}, k)$ were classified in [2] for $\mathfrak{g}=s l_{2}$ and in [17, [1] for general $\mathfrak{g}$, and the fusion rules were computed in [22] for $s l_{2}$ and in [1] for general $\mathfrak{g}$ with the help of quantum dimensions. See recent work in [6], [7], [19], [22], [3] and [28] for other topics on Parafermion vertex operator algebras.

The trace functions $Z_{M}(v, \tau)$ are the main objects in this paper where $M$ is an irreducible $K(\mathfrak{g}, k)$-module and $v \in K(\mathfrak{g}, k)$. Since $K(\mathfrak{g}, k)$ is rational and $C_{2}$-cofinite, the space spanned by $Z_{M}(v, \tau)$ for the irreducible modules $M$ affords a representation of the modular group $S L_{2}(\mathbb{Z})$ [36]. Our goal is to determine this representation explicitly. The irreducible $K(\mathfrak{g}, k)$-modules are labeled by $M^{\Lambda, \lambda}$. Here $\Lambda$ is a dominant weight of $\mathfrak{g}$ such that $\langle\Lambda, \theta\rangle \leq k,\langle$,$\rangle is the normalized invariant bilinear form on \mathfrak{g}$ so that the squared length of a long root is $2, \theta$ is the maximal root, $\lambda \in \Lambda+Q$ modulo $k Q_{L}$ and $Q$ is the root lattice. In the case $v=1$ the functions $\chi_{M^{\Lambda, \lambda}}(\tau)$ are a special kind of branching functions studied previously in [29], [30]. Moreover, $\chi_{M^{\Lambda, \lambda}}(\tau) / \eta(\tau)^{l}$ is the string function

The first author was supported by China NSF grant 11871351.

The third author was supported by China NSF grant 11671277. 
in [29], [30] where $l$ is the rank of $\mathfrak{g}$. In fact, explicit modular transformation formulas for branching functions were obtained in [29]. Our main result in this paper is that the same transformation formula for the branching functions is valid for the trace function with $\mathbf{1}$ replaced by any $v \in K(\mathfrak{g}, k)$.

The main idea is to use the modular transformation formulas for the affine vertex operator algebra $L_{\widehat{\mathfrak{g}}}(k, 0)$ and lattice vertex operator algebras $V_{\sqrt{k} Q_{L}}$. There are two modular transformation formulas for the affine vertex operator algebra $L_{\widehat{\mathfrak{g}}}(k, 0)$ given in [29] explicitly and [36] abstractly. Using a transformation formula for the abstract theta functions studied in [31], 35], one can easily show that these two transformation formulas are the same. Unfortunately, this can only give the modular transformation formula for the character $\chi_{M^{\Lambda, \lambda}}(\tau)$, not for the one point function $Z_{M^{\Lambda, \lambda}}(v, \tau)$. Again,results in [31] on abstract theta functions including some vector $w$ help us to solve the problem. To explain what kind of vector $w$ is, we recall from [16] that if $V=\oplus_{n \geq 0} V_{n}$ is a rational , $C_{2}$-cofinite, simple vertex operator algebra of CFT type, then $V_{1}$ is a reductive Lie algebra whose rank (the dimension of a Cartan subalgebra) is less than or equal to the central charge. Fix a Cartan subalgebra $\mathfrak{h}$ of $V_{1}$, vector $w$ satisfies conditions $h_{n} w=0$ for all $h \in \mathfrak{h}$ and $n \geq 0$. In the case $V=L_{\mathfrak{g}}(k, 0)$, the condition is exactly equivalent to $w \in K(\mathfrak{g}, k)$. The rest of the proof is similar to that given in [29] for the branching functions.

The paper is organized as follows: We review basics on vertex operator algebras and their modules in Section 2 including rationality, $C_{2}$-cofiniteness and modular transformation results on the trace functions associated to a rational and $C_{2}$-cofinite vertex operator algebra $V$. Following [35] and [31, we discuss Section 3 the modular transformation formula for generalized theta functions $\chi_{i}(w, v, q)=\operatorname{tr}_{M^{i}} O(w) e^{2 \pi i o(v)} q^{L(0)-c / 24}$ where $M^{i}$ is an irreducible $V$-module and $v \in \mathfrak{h}$ and $w$ is defined as before. In Section 4 we discuss the affine vertex operator algebras $L_{\widehat{\mathfrak{g}}}(k, 0)$ and the modular transformation formula of the specialized characters of the level $k$ integrable highest weight modules for the the affine Kac-Moody algebra $\widehat{\mathfrak{g}}$. We recall the various known results on the Parafermion vertex operator algebra $K(\mathfrak{g}, k)$ such as rationality and classification of irreducible modules. Section 6 deals with the modular transformation formula for the 1 point function $Z_{M^{\Lambda, \lambda}}(v, \tau)$. The $T$-matrix is easy and the most effort is on the $S$-matrix. The idea and method for finding the $S$-matrix is similar to that in [29] and [30].

\section{BASICS}

In this section we review the basic on vertex operator algebra. Let $V=(V, Y, \mathbf{1}, \omega)$ be a vertex operator algebra (cf. [4] and 25]).

A vertex operator algebra $V$ is of $C F T$ type if $V$ is simple, with respect to $L(0)$ one has $V=\oplus_{n \geq 0} V_{n}$ and $V_{0}=\mathbb{C} 1$ [12]. 
A vertex operator algebra $V$ is called $C_{2}$-cofinite if $\operatorname{dim} V / C_{2}(V)<\infty$ where $C_{2}(V)$ is the subspace of $V$ spanned by $u_{-2} v$ for $u, v \in V$ [36].

A weak $V$-module $M=\left(M, Y_{M}\right)$ is a vector space equipped with a linear map

$$
\begin{aligned}
Y_{M}: \quad V \rightarrow(\text { End } M)\left[\left[z^{-1}, z\right]\right] \\
v \mapsto Y_{M}(v, z)=\sum_{n \in \mathbb{Z}} v_{n} z^{-n-1} \quad\left(v_{n} \in \text { End } M\right) \text { for } v \in V
\end{aligned}
$$

satisfying the following conditions for $u, v \in V, w \in M$ :

$$
\begin{gathered}
v_{n} w=0 \quad \text { for } n \in \mathbb{Z} \text { sufficiently large; } \\
Y_{M}(\mathbf{1}, z)=1 ; \\
z_{0}^{-1} \delta\left(\frac{z_{1}-z_{2}}{z_{0}}\right) Y_{M}\left(u, z_{1}\right) Y_{M}\left(v, z_{2}\right)-z_{0}^{-1} \delta\left(\frac{z_{2}-z_{1}}{-z_{0}}\right) Y_{M}\left(v, z_{2}\right) Y_{M}\left(u, z_{1}\right) \\
=z_{2}^{-1} \delta\left(\frac{z_{1}-z_{0}}{z_{2}}\right) Y_{M}\left(Y\left(u, z_{0}\right) v, z_{2}\right) .
\end{gathered}
$$

An (ordinary) $V$-module is a weak $V$-module $M$ which is $\mathbb{C}$-graded

$$
M=\bigoplus_{\lambda \in \mathbb{C}} M_{\lambda}
$$

such that $\operatorname{dim} M_{\lambda}$ is finite and $M_{\lambda+n}=0$ for fixed $\lambda$ and $n \in \mathbb{Z}$ small enough, where $M_{\lambda}$ is the eigenspace for $L(0)$ with eigenvalue $\lambda$ :

$$
L(0) w=\lambda w, \quad w \in M_{\lambda} .
$$

Let $M$ be an ordinary $V$-module. We denote $v_{n-1}$ by $o(v)$ for $v \in V_{n}$ and extend to $V$ linearly. Then $o(v) M_{\lambda} \subset M_{\lambda}$ for all $\lambda \in \mathbb{C}$.

An admissible $V$-module is a weak $V$-module $M$ which carries a $\mathbb{Z}_{+}$-grading

$$
M=\bigoplus_{n \in \mathbb{Z}_{+}} M(n)
$$

$\left(\mathbb{Z}_{+}\right.$is the set all nonnegative integers) such that if $r, m \in \mathbb{Z}, n \in \mathbb{Z}_{+}$and $a \in V_{r}$ then

$$
a_{m} M(n) \subseteq M(r+n-m-1) .
$$

Note that any ordinary module is an admissible module.

A vertex operator algebra $V$ is called rational if any admissible module is a direct sum of irreducible admissible modules [9].

The following result is proved in [10] and [11].

Theorem 2.1. Assume that $V$ is rational. 
(1) There are only finitely many inequivalent irreducible admissible modules $V=M^{0}, \ldots, M^{p}$ and each irreducible admissible module is an ordinary module. Each $M^{i}$ has weight space decomposition

$$
M^{i}=\bigoplus_{n \geq 0} M_{\lambda_{i}+n}^{i}
$$

where $\lambda_{i} \in \mathbb{C}$ is a complex number such that $M_{\lambda_{i}}^{i} \neq 0$ and $M_{\lambda_{i}+n}^{i}$ is the eigenspace of $L(0)$ with eigenvalue $\lambda_{i}+n$. The $\lambda_{i}$ is called the weight of $M^{i}$.

(2) If $V$ is both rational and $C_{2}$-cofinite, then $\lambda_{i}$ and central charge $c$ are rational numbers.

In the rest of this paper we assume that $V$ is a strong rational vertex operator algebra. That is $V$ satisfies the following:

(V1) $V=\oplus_{n \geq 0} V_{n}$ is a simple vertex operator algebra of CFT type,

(V2) $V$ is $C_{2}$-cofinite and rational,

(V3) The conformal weights $\lambda_{i}$ are nonnegative and $\lambda_{i}=0$ if and only if $i=0$.

Using the assumption (V3) we know that $V$ and its contragredient module $V^{\prime}$ [24] are isomorphic $V$-modules. From [16] we know $V_{1}$ is a reductive Lie algebra with $[u, v]=u_{0} v$ for $u, v \in V_{1}$. Moreover, the rank $V_{1}$ is less than or equal to the central charge $c$ of $V$. For any $u \in V_{1}, u_{0}$ is a derivation of $V$ in the sense that $\left[u_{0}, Y(v, z)\right]=Y\left(u_{0} v, z\right)$ for any $v \in V$ and $u_{0} \omega=0$. Furthermore, $e^{u_{0}}$ is an automorphism of $V$.

Recall from 24 that a bilinear from $(\cdot, \cdot)$ on $V$ is called invariant if

$$
(Y(u, z) v, w)=\left(u, Y\left(e^{z L(1)}\left(-z^{-2}\right)^{L(0)} v, z^{-1}\right) w\right)
$$

for $u, v, w \in V$. From our assumptions there is unique nondegenerate invariant bilinear form on $V$ [33]. We shall fix a bilinear form $(\cdot, \cdot)$ on $V$ so that $(u, v)=u_{1} v$ for $u, v \in V_{1}$. It is clear from the definition that $(g u, g v)=(u, v)$ for any automorphism $g$ and $u, v \in V_{1}$.

The modular transformations of trace functions of irreducible modules of vertex operator algebras [36] are of main importance in this paper. Another vertex operator algebra structure $(V, Y[\cdot, z], \mathbf{1}, \omega-c / 24)$ is defined on $V$ in [36] with grading

$$
V=\bigoplus_{n \geq 0} V_{[n]}
$$

For $v \in V_{[n]}$ we write wt $[v]=n$. For $i=0, \ldots, p$ we set

$$
Z_{i}(v, q)=\operatorname{tr}_{M^{i} O}(v) q^{L(0)-c / 24}
$$

which is a formal power series in variable $q$. The $Z_{i}(\mathbf{1}, q)$ which is also denoted by $\operatorname{ch}_{q} M^{i}$ is called the $q$-character of $M^{i}$.

The series $Z_{i}(v, q)$ converges to a holomorphic function $Z_{i}(v, \tau)$ on $\mathbb{H}=\{\tau \in \mathbb{C} \mid \operatorname{Im} \tau>$ $0\}$ where $q=e^{2 \pi i \tau}$ with $\tau \in \mathbb{H}[36]$. 
Recall the modular group $S L_{2}(\mathbb{Z})$ and let $\gamma=\left(\begin{array}{ll}a & b \\ c & d\end{array}\right) \in S L_{2}(\mathbb{Z})$.

Theorem 2.2. Let $V$ be a strong rational vertex operator algebra.

(1) There is a group homomorphism $\rho: S L_{2}(\mathbb{Z}) \rightarrow G L_{p+1}(\mathbb{C})$ with $\rho(\gamma)=\left(\gamma_{i j}\right)$ such that for any $0 \leq i \leq p$ and $v \in V$,

$$
Z_{i}\left(v, \frac{a \tau+b}{c \tau+d}\right)=(c \tau+d)^{\mathrm{wt}[v]} \sum_{j=0}^{p} \gamma_{i j} Z_{j}(v, \tau)
$$

(2) Each $Z_{i}(v, \tau)$ is a modular form of weight $\mathrm{wt}[v]$ over a congruence subgroup.

Part (1) of the Theorem was obtained in [36] and Part (2) was established in [13]. The matrices

$$
S=\rho_{V}\left(\left[\begin{array}{cc}
0 & -1 \\
1 & 0
\end{array}\right]\right) \text { and } T=\rho_{V}\left(\left[\begin{array}{ll}
1 & 1 \\
0 & 1
\end{array}\right]\right)
$$

are respectively called the genus one $S$ - and $T$-matrices of $V$.

\section{Modular inVARIANCE OF THE GENERALIZED THETA FUNCTIONS}

We review the modular transformation formula of the theta functions defined on vertex operator algebra given in [35] and [31] for studying the modular invariance of trace functions for the parafermion vertex operator algebras.

Recall that $V_{1}$ is a reductive Lie algebra. We fix a Cartan subalgebra $\mathfrak{h}$ of $V_{1}$. Then the abelian Lie algebra $\mathfrak{h}$ acts on $M^{i}$ semsimply for all $i$. Following [35, we define the generalized theta functions as

$$
T_{i}(v, u, q)=\operatorname{tr}_{M^{i}} e^{2 \pi i\left(v_{0}+(u, v) / 2\right)} q^{L(0)+u_{0}+(u, u) / 2-c / 24}
$$

for $u, v \in \mathfrak{h}$. The bilinear form on $V_{1}$ used in [35] is the negative of the bilinear form used in this paper. So our $T_{i}(v, u, q)$ defined here is exactly the $Z_{M^{i}}(v, u, q)$ in [35]. Based on Theorem 2.2, a modular transformation law was obtained in [35] with the convergence of $Z_{i}(v, u, q)$ proved in [14].

Theorem 3.1. Let $u, v \in \mathfrak{h}$ and $\gamma=\left(\begin{array}{ll}a & b \\ c & d\end{array}\right) \in S L_{2}(\mathbb{Z})$. Then $T_{i}(v, u, q)$ converges to a holomorphic function in the upper half plane with $q=e^{2 \pi i \tau}$ and

$$
T_{i}\left(v, u, \frac{a \tau+b}{c \tau+d}\right)=\sum_{j=0}^{p} \gamma_{i j} T_{j}(d v+b u, c v+a u, \tau)
$$

where $\gamma_{i j}$ is the same as in Theorem 2.2.

Set $\chi_{i}(v, \tau)=T_{i}(v, 0, \tau)$. Using Theorem 3.1 one can easily show the following result [14]. 
Proposition 3.2. Assume that $V$ is a strong rational vertex operator algebra. Then for $\gamma=\left(\begin{array}{ll}a & b \\ c & d\end{array}\right) \in S L_{2}(\mathbb{Z}), v \in \mathfrak{h}$

$$
\chi_{s}\left(\frac{v}{c \tau+d}, \frac{a \tau+b}{c \tau+d}\right)=e^{\pi i(c(v, v) /(c \tau+d))} \sum_{j=0}^{p} \gamma_{s j} \chi_{j}(v, \tau) .
$$

As usual we set $o(w)=w_{\mathrm{wt} w-1}$ for homogeneous $w \in V$ and extend to whole $V$. Now take $w \in V$ such that $h_{n} w=0$ for all $h \in \mathfrak{h}$ and $n \geq 0$. Also define

$$
\chi_{i}(w, v, q)=\operatorname{tr}_{M^{i}} O(w) e^{2 \pi i o(v)} q^{L(0)-c / 24} .
$$

The following result [31] generalizes Proposition 3.2 .

Theorem 3.3. Let $v \in \mathfrak{h}$. We assume that $\chi_{i}(w, v, q)$ converges to a holomorphic function $\chi_{i}(w, v, \tau)$ in $\mathbb{H}$ with $q=e^{2 \pi i \tau}$ for any $v \in \mathfrak{h}$. Then for $\gamma=\left(\begin{array}{ll}a & b \\ c & d\end{array}\right) \in S L_{2}(\mathbb{Z})$,

$$
\chi_{i}\left(w, \frac{v}{c \tau+d}, \frac{a \tau+b}{c \tau+d}\right)=(c \tau+d)^{\mathrm{wt}[v]} e^{\frac{\pi i c(v, v)}{c \tau+d}} \sum_{j=0}^{p} \gamma_{i j} \chi_{j}(w, v, \tau) .
$$

\section{Affine vertex operator Algebras}

In this section we discuss the affine vertex operator algebra $L_{\widehat{\mathfrak{g}}}(k, 0)$ associated to the level $k$ integrable highest weight module for affine Kac-Moody algebra $\widehat{\mathfrak{g}}$ and its irreducible modules.

Let $\mathfrak{g}$ be a finite dimensional simple Lie algebra with a Cartan subalgebra $\mathfrak{h}$. We denote the corresponding root system by $\Delta$ and the root lattice by $Q$. Fix an invariant symmetric nondegenerate bilinear form $\langle$,$\rangle on \mathfrak{g}$ such that $\langle\alpha, \alpha\rangle=2$ if $\alpha$ is a long root, where we have identified $\mathfrak{h}$ with $\mathfrak{h}^{*}$ via $\langle$,$\rangle . We denote the image of \alpha \in \mathfrak{h}^{*}$ in $\mathfrak{h}$ by $t_{\alpha}$. That is, $\alpha(h)=\left\langle t_{\alpha}, h\right\rangle$ for any $h \in \mathfrak{h}$. Fix simple roots $\left\{\alpha_{1}, \ldots, \alpha_{l}\right\}$ and let $\Delta_{+}$be the set of corresponding positive roots. Denote the highest root by $\theta$, and the Weyl group by $W$. Also let $\rho$ be the half sum of positive roots.

Recall that the weight lattice $P$ of $\mathfrak{g}$ consists of $\lambda \in \mathfrak{h}^{*}$ such that $\frac{2\langle\lambda, \alpha\rangle}{\langle\alpha, \alpha\rangle} \in \mathbb{Z}$ for all $\alpha \in \Delta$. It is well-known that $P=\bigoplus_{i=1}^{l} \mathbb{Z} \Lambda_{i}$ where $\Lambda_{i}$ are the fundamental weights defined by the equation $\frac{2\left\langle\Lambda_{i}, \alpha_{j}\right\rangle}{\left\langle\alpha_{j}, \alpha_{j}\right\rangle}=\delta_{i, j}$ for $1 \leq i, j \leq l$. Let $P_{+}$be the subset of $P$ consisting of the dominant weight $\Lambda \in P$ in the sense that $\frac{2\left\langle\Lambda, \alpha_{j}\right\rangle}{\left\langle\alpha_{j}, \alpha_{j}\right\rangle}$ is nonnegative for all $j$. For any nonnegative integer $k$ we also let $P_{+}^{k}$ be the subset of $P_{+}$consisting of $\Lambda$ satisfying $\langle\Lambda, \theta\rangle \leq k$.

Let $Q=\sum_{i=1}^{l} \mathbb{Z} \alpha_{i}$ be the root lattice and $Q_{L}$ be the sublattice of $Q$ spanned by the long roots. Recall that the dual lattice $Q_{L}^{\circ}$ consists $\lambda \in \mathfrak{h}^{*}$ such that $\langle\lambda, \alpha\rangle \in \mathbb{Z}$ for all $\alpha \in Q_{L}$. Then $P$ is the dual lattice of $Q_{L}$ [1]. 
Let $\widehat{\mathfrak{g}}=\mathfrak{g} \otimes \mathbb{C}\left[t, t^{-1}\right] \oplus \mathbb{C} K$ be the affine Lie algebra. Fix a nonnegative integer $k$. For any $\Lambda \in P_{+}^{k}$ let $L(\Lambda)$ be the irreducible highest weight $\mathfrak{g}$-module with highest weight $\Lambda$ and $L_{\widehat{\mathfrak{g}}}(k, \Lambda)$ be the unique irreducible $\widehat{\mathfrak{g}}$-module such that $L_{\widehat{\mathfrak{g}}}(k, \Lambda)$ is generated by $L(\Lambda)$ and $\mathfrak{g} \otimes t^{n} L(\Lambda)=0$ for $t>0$ and $K$ acts as constant $k$. The following result is well known (cf. [26], [32]):

Theorem 4.1. The $L_{\mathfrak{\mathfrak { g }}}(k, 0)$ is a simple, rational and $C_{2}$-cofinite vertex operator algebra with central charge $\frac{k \operatorname{dimg}}{k+h^{\vee}}$ and whose irreducible modules are $L_{\widehat{\mathfrak{g}}}(k, \Lambda)$ for $\Lambda \in P_{+}^{k}$ and the weight $n_{\Lambda}$ of $L_{\widehat{\mathfrak{g}}}(k, \Lambda)$ is $\frac{\langle\Lambda+2 \rho, \Lambda\rangle}{2\left(k+h^{\vee}\right)}$ where $\rho=\sum_{i=1}^{l} \Lambda_{i}$ and $h^{\vee}$ is the dual Coxeter number.

Note that $L_{\widehat{\mathfrak{g}}}(k, \Lambda)$ has a decomposition with respect to the action of $\mathfrak{h}$ :

$$
L_{\widehat{\mathfrak{g}}}(k, \Lambda)=\oplus_{\lambda \in \Lambda+Q} L_{\widehat{\mathfrak{g}}}(k, \Lambda)(\lambda)
$$

where $h(0)$ acts on $L_{\widehat{\mathfrak{g}}}(k, \Lambda)(\lambda)$ as constant $\lambda(h)=\langle\lambda, h\rangle$ for $h \in \mathfrak{h}$. Following [29], define the character

$$
\chi_{\Lambda}(h, \tau)=\operatorname{tr}_{L_{\widehat{\mathfrak{g}}}(k, \Lambda)} e^{2 \pi i h(0)} q^{L(0)-c / 24}
$$

for $h \in \mathfrak{h}$. Note that the character defined in [29] has an extra factor $e^{2 \pi i k u}$ with $u$ being a complex number. This extra factor makes the modular transformation formula more beautiful. But from the point of view of vertex operator algebra, this extra factor is not necessary. From [29] we have

Theorem 4.2. Let $\mathfrak{g}$ and $k$ be as before.

(1) $\left\{\chi_{\Lambda}(h, \tau) \mid \Lambda \in P_{+}^{k}\right\}$ are linearly independent functions on $\mathfrak{h} \times \mathbb{H}$.

(2) For $\Lambda, \Lambda^{\prime} \in P_{+}^{k}$, set

$$
S_{\Lambda, \Lambda^{\prime}}=i^{\left|\Delta_{+}\right|}\left|P /\left(k+h^{\vee}\right) Q_{L}\right|^{-1 / 2} \sum_{w \in W}(-1)^{l(w)} e^{-\frac{2 \pi i}{k+h^{\vee}}\left\langle w(\Lambda+\rho), \Lambda^{\prime}+\rho\right\rangle}
$$

where $l(w)$ is the length of $w$. Then

$$
\chi_{\Lambda}\left(\frac{h}{\tau}, \frac{-1}{\tau}\right)=e^{\pi i k\langle h, h\rangle / \tau} \sum_{\Lambda^{\prime} \in P_{+}^{k}} S_{\Lambda, \Lambda^{\prime}} \chi_{\Lambda^{\prime}}(h, \tau) .
$$

Recall Theorem 2.2 and Proposition 3.2.

Lemma 4.3. The S-matrices in Theorem 2.2 and Theorem 4.2 are the same.

Proof. Let $V=L_{\widehat{\mathfrak{g}}}(k, 0)$. Then $V$ satisfies the assumptions in Theorem 2.2. Recall that we have defined a bilinear form on $V_{1}$ such that $(u, v)=u_{1} v$. Let $u=a(-1) \mathbf{1}$ and $v=b(-1) \mathbf{1}$ for $a, b \in \mathfrak{g}$. Then $(u, v)=a(1) b(-1) \mathbf{1}=k\langle a, b\rangle$. Identify Lie algebra $V_{1}$ with $\mathfrak{g}$ we see 
that $(a, b)=k\langle a, b\rangle$. We denote the $S$-matrix in Theorem 2.2 by $\left(S_{L_{\widehat{\mathfrak{g}}}(k, \Lambda), L_{\widehat{\mathfrak{g}}}\left(k, \Lambda^{\prime}\right)}\right)$. Then from Proposition 3.2 we know

$$
\chi_{\Lambda}\left(\frac{h}{\tau}, \frac{-1}{\tau}\right)=e^{\pi i k\langle h, h\rangle / \tau} \sum_{\Lambda^{\prime} \in P_{+}^{k}} S_{L_{\widehat{\mathfrak{g}}}(k, \Lambda), L_{\widehat{\mathfrak{g}}}\left(k, \Lambda^{\prime}\right)} \chi_{\Lambda^{\prime}}(h, \tau) .
$$

The result now is an immediate consequence of Theorem 4.2 (1).

\section{PARAFERMion Vertex operator Algebras}

In this section we recall the definition of a parafermion vertex operator algebra $K(\mathfrak{g}, k)$ associated to any finite dimensional simple Lie algebra $\mathfrak{g}$ and a positive integer $k$. We also discuss some known results on $K(\mathfrak{g}, k)$ from [17].

Let $\lambda_{i} \in P$ such that $\lambda_{i}=\frac{\langle\theta, \theta\rangle}{\left\langle\alpha_{i}, \alpha_{i}\right\rangle} \Lambda_{i}$ for $i=1, \ldots, l$. Then $\left\langle\alpha_{i}, \lambda_{j}\right\rangle=\delta_{i, j}$ for all $i, j$ and $Q^{\circ}=\bigoplus_{i=1}^{l} \mathbb{Z} \lambda_{i}$. The following result is immediate from the relation between $\Lambda_{i}$ and $\lambda_{i}$.

Lemma 5.1. $P / Q^{\circ}$ is a group of order

$$
\left|P / Q^{\circ}\right|= \begin{cases}1 & A_{l}, D_{l}, E_{6}, E_{7}, E_{8} \\ 2 & B_{l} \\ 2^{l-1} & C_{l} \\ 2^{2} & F_{4} \\ 3 & G_{2}\end{cases}
$$

Lemma 5.2. For any simple Lie algebra $\mathfrak{g}$ and any positive integer $k, \frac{1}{k} P / Q^{\circ}$ and $Q / k Q_{L}$ are dual groups, so that for any $\beta \in Q, g_{\beta}(\alpha)=e^{2 \pi i\langle\beta, \alpha\rangle}$ defines an irreducible character for $\frac{1}{k} P / Q^{\circ}$ In particular, $\frac{1}{k} P / Q^{\circ}$ and $Q / k Q_{L}$ are isomorphic groups.

Proof. Clearly, $g_{\beta}$ defines a irreducible character of $\frac{1}{k} P / Q^{\circ}$ as $Q_{L}^{\circ}=P$. Also, $g_{\beta_{1}}=g_{\beta_{2}}$ for if and only if $\beta_{1}-\beta_{2} \in k Q_{L}$. So $Q / k Q_{L}$ is a subgroup of the dual group of $\frac{1}{k} P / Q^{\circ}$. To finish the proof, it is enough to show that $\left|P / Q^{\circ}\right|=\left|Q / Q_{L}\right|$. This is obvious if $\mathfrak{g}$ is a Lie algebra of type $A, D, E$. If $\mathfrak{g}$ is a Lie algebra of other type, we verify the result case by case using the root systems given in [27]. We have already known $\left|P / Q^{\circ}\right|$ from Lemma 5.1. So we only need to compute $\left|Q / Q_{L}\right|$.

(1) Type $B_{l}$. Let $\mathbb{E}=\mathbb{R}^{l}$ with the standard orthonormal basis $\left\{\epsilon_{1}, \ldots, \epsilon_{l}\right\}$. Then

$$
\Delta=\left\{ \pm \epsilon_{i}, \pm\left(\epsilon_{i} \pm \epsilon_{j}\right) \mid i \neq j\right\} .
$$

Then $Q=\sum_{i}^{l} \mathbb{Z} \epsilon_{i}$ and $Q_{L}=\sum_{i \neq j}\left(\mathbb{Z}\left(\epsilon_{i}+\epsilon_{j}\right)+\mathbb{Z}\left(\epsilon_{i}-\epsilon_{j}\right)\right)$. It is evident that $2 Q \subset Q_{L}$ and $\left|Q / Q_{L}\right|=2$ with coset representatives 0 and $\epsilon_{1}$.

(2) Type $C_{l}$. In this case,

$$
\Delta=\left\{ \pm \sqrt{2} \epsilon_{i}, \pm \frac{1}{\sqrt{2}}\left(\epsilon_{i} \pm \epsilon_{j}\right) \mid i \neq j\right\}
$$


Then $Q=\frac{1}{\sqrt{2}} \sum_{i \neq j}\left(\mathbb{Z}\left(\epsilon_{i}+\epsilon_{j}\right)+\mathbb{Z}\left(\epsilon_{i}-\epsilon_{j}\right)\right)$ and $Q_{L}=\sqrt{2} \sum_{i=1}^{l} \mathbb{Z} \epsilon_{i}$. Thus $\left|Q / Q_{L}\right|=2^{l-1}$ with coset representatives $a_{1} \alpha_{1}+a_{2} \alpha_{2}+\cdots+a_{l-1} \alpha_{l-1}$ for $a_{i}=0,1$ and $\alpha_{i}=\frac{1}{\sqrt{2}}\left(\epsilon_{i}-\epsilon_{i+1}\right)$.

(3) Type $F_{4}$. Let $\mathbb{E}=\mathbb{R}^{4}$. Then

$$
\Delta=\left\{ \pm \epsilon_{i}, \pm\left(\epsilon_{i} \pm \epsilon_{j}\right), \pm \frac{1}{2}\left(\epsilon_{1} \pm \epsilon_{2} \pm \epsilon_{3} \pm \epsilon_{4}\right) \mid i \neq j\right\} .
$$

Then $\left|Q / Q_{L}\right|=2^{2}$ with coset representatives $a \epsilon_{3}+b \frac{1}{2}\left(\epsilon_{1}-\epsilon_{2}-\epsilon_{3}-\epsilon_{4}\right)$ for $a, b=0,1$.

(4) Type $G_{2}$. Let $\mathbb{E}$ be the subspace of $\mathbb{R}^{3}$ orthogonal to $\epsilon_{1}+\epsilon_{2}+\epsilon_{3}$. Then

$$
\Delta= \pm \frac{1}{\sqrt{3}}\left\{\epsilon_{i}-\epsilon_{j}, 2 \epsilon_{1}-\epsilon_{2}-\epsilon_{3}, 2 \epsilon_{2}-\epsilon_{1}-\epsilon_{3}, 2 \epsilon_{3}-\epsilon_{1}-\epsilon_{2} \mid i \neq j\right\} .
$$

Then $\left|Q / Q_{L}\right|=3$ with coset representatives $a \frac{1}{\sqrt{3}}\left(\epsilon_{1}-\epsilon_{2}\right)$ for $a=0,1,2$. The proof is complete.

Let $M_{\widehat{\mathfrak{h}}}(k)$ be the vertex operator subalgebra of $L_{\widehat{\mathfrak{g}}}(k, 0)$ generated by $h(-1) \mathbf{1}$ for $h \in$ $\mathfrak{h}$. For $\lambda \in \mathfrak{h}^{*}$, denote by $M_{\widehat{\mathfrak{h}}}(k, \lambda)$ the irreducible highest weight module for $\widehat{\mathfrak{h}}$ with a highest weight vector $e^{\lambda}$ such that $h(0) e^{\lambda}=\lambda(h) e^{\lambda}$ for $h \in \mathfrak{h}$. The parafermion vertex operator algebra $K(\mathfrak{g}, k)$ is the commutant [26] of $M_{\widehat{\mathfrak{h}}}(k)$ in $L_{\widehat{\mathfrak{g}}}(k, 0)$. We have the following decomposition

$$
L_{\widehat{\mathfrak{g}}}(k, \Lambda)=\bigoplus_{\lambda \in Q+\Lambda} M_{\widehat{\mathfrak{h}}}(k, \lambda) \otimes M^{\Lambda, \lambda}=\bigoplus_{\alpha \in Q} M_{\widehat{\mathfrak{h}}}(k, \Lambda+\alpha) \otimes M^{\Lambda, \Lambda+\alpha}
$$

as $M_{\widehat{\mathfrak{h}}}(k) \otimes K(\mathfrak{g}, k)$-module. Moreover, $M^{0,0}=K(\mathfrak{g}, k)$ and $M^{\Lambda, \lambda}$ is an irreducible $K(\mathfrak{g}, k)$ module [17]. Recall equation (5.1). It is easy to see that

$$
L_{\widehat{\mathfrak{g}}}(k, \Lambda)(\lambda)=M_{\widehat{\mathfrak{h}}}(k, \lambda) \otimes M^{\Lambda, \lambda} .
$$

It is proved in [21] that the lattice vertex operator algebra $V_{\sqrt{k} Q_{L}}$ is a vertex operator subalgebra of $L_{\widehat{\mathfrak{g}}}(k, 0)$ and the parafermion vertex operator algebra $K(\mathfrak{g}, k)$ is also a commutant of $V_{\sqrt{k} Q_{L}}$ in $L_{\widehat{\mathfrak{g}}}(k, 0)$. This gives us another decomposition

$$
L_{\widehat{\mathfrak{g}}}(k, \Lambda)=\bigoplus_{i \in Q / k Q_{L}} V_{\sqrt{k} Q_{L}+\frac{1}{\sqrt{k}}\left(\Lambda+\beta_{i}\right)} \otimes M^{\Lambda, \Lambda+\beta_{i}}
$$

as modules for $V_{\sqrt{k} Q_{L}} \otimes K(\mathfrak{g}, k)$, where $M^{\Lambda, \lambda}$ is as before, $Q=\cup_{i \in Q / k Q_{L}}\left(k Q_{L}+\beta_{i}\right)$, and $\beta_{i} \in Q$ is a representative of $i$. Moreover, for any $i \in Q / k Q_{L}$, we have

$$
V_{\sqrt{k} Q_{L}+\frac{1}{\sqrt{k}}\left(\Lambda+\beta_{i}\right)} \otimes M^{\Lambda, \Lambda+\beta_{i}}=\bigoplus_{\alpha \in k Q_{L}} M_{\widehat{\mathfrak{h}}}\left(k, \Lambda+\alpha+\beta_{i}\right) \otimes M^{\Lambda, \Lambda+\alpha+\beta_{i}}
$$

where we have used the isomorphism between $K(\mathfrak{g}, k)$-modules $M^{\Lambda, \Lambda+\alpha+\beta_{i}}$ and $M^{\Lambda, \Lambda+\beta_{i}}$ for any $\alpha \in k Q_{L}$ [17]. So for any $h \in \mathfrak{h}, h(0) \in \widehat{\mathfrak{g}}$ acts as $\sqrt{k} h(0)$ or $\left\langle h, k \alpha+\Lambda+\beta_{i}\right\rangle$ on $e^{\sqrt{k} \alpha+\frac{1}{\sqrt{k}}\left(\Lambda+\beta_{i}\right)}$.

Let $\theta=\sum_{i=1}^{l} a_{i} \alpha_{i}$. According to [33], 34], if $a_{i}=1$ then $L_{\widehat{\mathfrak{g}}}\left(k, k \Lambda_{i}\right)$ is a simple current and $L_{\widehat{\mathfrak{g}}}\left(k, k \Lambda_{i}\right) \otimes L_{\widehat{\mathfrak{g}}}(k, \Lambda)=L_{\widehat{\mathfrak{g}}}\left(k, \Lambda^{(i)}\right)$ for any $\Lambda \in P_{+}^{k}$ where $\Lambda^{(i)} \in P_{+}^{k}$ is uniquely 
determined by $\Lambda$ and $i$. Then $L_{\widehat{\mathfrak{g}}}(k, \Lambda)$ and $L_{\widehat{\mathfrak{g}}}\left(k, \Lambda^{(i)}\right)$ are isomorphic $K(\mathfrak{g}, k)$-modules [17.

Here are some main results on $K(\mathfrak{g}, k)$ from [20, 2], 3], 17], [1].

Theorem 5.3. Let $\mathfrak{g}$ be a simple Lie algebra and $k$ a positive integer.

(1) The $K(\mathfrak{g}, k)$ is a rational, simple and $C_{2}$-cofinite vertex operator algebra of $C F T$ type.

(2) For any $\Lambda \in P_{+}^{k}, \lambda \in \Lambda+Q$ and $\alpha \in Q_{L}, M^{\Lambda, \lambda}=M^{\Lambda, \lambda+k \alpha}$.

(3) For each $i \in I, \Lambda \in P_{+}^{k}$ there exists a unique $\Lambda^{(i)} \in P_{+}^{k}$ such that for any $\lambda \in \Lambda+Q$, $M^{\Lambda, \lambda}=M^{\Lambda^{(i)}, \lambda+k \Lambda_{i}}$.

(4) Any irreducible $K(\mathfrak{g}, k)$-module is isomorphic to $M^{\Lambda, \lambda}$ for some $\Lambda \in P_{+}^{k}$ and $\lambda \in$ $\Lambda+Q$.

(5) The $K(\mathfrak{g}, k)$ has exactly $\frac{\left|P_{+}^{k}\right|\left|Q / k Q_{L}\right|}{|P / Q|}$ inequivalent irreducible modules.

\section{TRACE FUnCtions FOR PARAFERMion VERTEX OPERATOR ALGEBRAS}

In this section we determine the $S$-matrix for parafermion vertex operator algebra $K(\mathfrak{g}, k)$ associated to any finite dimensional simple Lie algebra $\mathfrak{g}$ and positive integer $k$ from [17].

From decomposition (5.1) we have

$$
\chi_{L_{\widehat{\mathfrak{g}}}(k, \Lambda)}(\tau)=\sum_{\lambda \in Q+\Lambda} \chi_{M_{\widehat{\mathfrak{h}}}(k, \lambda)}(\tau) \chi_{M^{\Lambda, \lambda}}(\tau)=\sum_{\lambda \in Q+\Lambda} \frac{q^{\langle\lambda, \lambda\rangle / 2 k}}{\eta(\tau)^{l}} \chi_{M^{\Lambda, \lambda}}(\tau)
$$

where $\eta(\tau)=q^{1 / 24} \prod_{n \geq 1}\left(1-q^{n}\right)$. The

$$
c_{\lambda}^{\Lambda}(\tau)=\eta(\tau)^{-l} \chi_{M^{\Lambda, \lambda}}(\tau)
$$

is called the string function and $\chi_{M^{\Lambda, \lambda}}(\tau)$ is called the branching function denoted by $b_{\lambda}^{\Lambda}(\tau)$ in [29]. The modular transformation formulas of branching functions were also given in [29]:

Theorem 6.1. Let $\Lambda \in P_{+}^{k}$ and $i \in Q / k Q_{L}$. Then

(1) $\chi_{M^{\Lambda, \Lambda+\beta_{i}}}(\tau+1)=e^{2 \pi i\left(\frac{\langle\Lambda+2 \rho, \Lambda\rangle}{2(k+h V)}-\frac{\left\langle\Lambda+\beta_{i}, \Lambda+\beta_{i}\right\rangle}{2 k}-\frac{k \operatorname{dimg}}{24\left(k+h^{V}\right)}+\frac{l}{24}\right)} \chi_{M^{\Lambda, \Lambda+\beta_{i}}}(\tau)$,

(2) $\chi_{M^{\Lambda, \Lambda+\beta_{i}}}\left(\frac{-1}{\tau}\right)=\sum_{\Lambda^{\prime} \in P_{+}^{k}, j \in Q / k Q_{L}} S_{\left(\Lambda, \Lambda+\beta_{i}\right),\left(\Lambda^{\prime}, \Lambda^{\prime}+\beta_{i}\right)}^{P} \chi_{M^{\Lambda^{\prime}, \Lambda^{\prime}+\beta_{j}}}(\tau)$, where

$$
S_{\left(\Lambda, \Lambda+\beta_{i}\right),\left(\Lambda^{\prime}, \Lambda^{\prime}+\beta_{i}\right)}^{P}=\left|P / k Q_{L}\right|^{-1 / 2} S_{\Lambda, \Lambda^{\prime}} e^{2 \pi i \frac{\left\langle\Lambda+\beta_{i}, \Lambda^{\prime}+\beta_{j}\right\rangle}{k}}
$$

and $P$ stands for Parafermoin.

The main result in this paper is that the transformation formula for the branching functions remains valid for the trace functions $Z_{M^{\Lambda, \lambda}}(w, \tau)$ with $w \in K(\mathfrak{g}, k)$ : 
Theorem 6.2. Let $\Lambda \in P_{+}^{k}$ and $i \in Q / k Q_{L}$. Then for any $w \in K(\mathfrak{g}, k)$

(1) $Z_{M^{\Lambda, \Lambda+\beta_{i}}}(w, \tau+1)=e^{2 \pi i\left(\frac{\langle\Lambda+2 \rho, \Lambda\rangle}{2(k+h \text { V }}-\frac{\left\langle\Lambda+\beta_{i}, \Lambda+\beta_{i}\right\rangle}{2 k}-\frac{k \operatorname{dim} \mathfrak{g}}{24\left(k+h^{V}\right)}+\frac{l}{24}\right)} Z_{M^{\Lambda, \Lambda+\beta_{i}}}(w, \tau)$,

(2) $Z_{M^{\Lambda, \Lambda+\beta_{i}}}\left(w, \frac{-1}{\tau}\right)=\tau^{\mathrm{wt}[w]} \sum_{\Lambda^{\prime} \in P_{+}^{k}, j \in Q / k Q_{L}} S_{\left(\Lambda, \Lambda+\beta_{i}\right),\left(\Lambda^{\prime}, \Lambda^{\prime}+\beta_{i}\right)}^{P} Z_{M^{\Lambda^{\prime}, \Lambda^{\prime}+\beta_{j}}}(w, \tau)$.

Proof. (1) is straightforward. The proof (2) is divided in several steps.

(a) $\chi_{L_{\widehat{\mathfrak{g}}}(k, \Lambda)}(w, h, q)$ converges to a holomorphic function $\chi_{L_{\widehat{\mathfrak{g}}}(k, \Lambda)}(w, h, \tau)$ for any $w \in$ $K(\mathfrak{g}, k)$ and $h \in \mathfrak{h}$ where we have identified $x \in \mathfrak{g}$ with $x(-1) \mathbf{1} \in L_{\widehat{\mathfrak{g}}}(k, 0)_{1}$.

We denote the Virasoro vectors of $L_{\widehat{\mathfrak{g}}}(k, 0), V_{\sqrt{k} Q_{L}}$ and $K(\mathfrak{g}, k)$ by $\omega^{a}, \omega^{l}$ and $\omega^{p}$ and denote the corresponding components of the vertex operators by $L^{a}(n), L^{l}(n)$ and $L^{p}(n)$ respectively for $n \in \mathbb{Z}$. Also denote the Virasoro central charges of $L_{\mathfrak{\mathfrak { g }}}(k, 0)$ and $K(\mathfrak{g}, k)$ by $c^{a}$ and $c^{p}$ respectively. Then $c^{a}=l+c^{p}$.

Recall decomposition (5.3). Then

$$
\begin{aligned}
& \chi_{L_{\widehat{\mathfrak{g}}}(k, \Lambda)}(w, h, q)=\operatorname{tr}_{L_{\widehat{\mathfrak{g}}}(k, \Lambda)} O(w) e^{2 \pi i h(0)} q^{L^{a}(0)-c^{a} / 24} \\
& =\sum_{i \in Q / k Q_{L}} \operatorname{tr}_{V_{\sqrt{k} Q_{L}+\frac{1}{\sqrt{k}}\left(\Lambda+\beta_{i}\right) \otimes M^{\Lambda, \Lambda+\beta_{i}}} O(w) e^{2 \pi i h(0)} q^{L^{a}(0)-c^{a} / 24}} \\
& =\sum_{i \in Q / k Q_{L}} \operatorname{tr}_{V_{\sqrt{k} Q_{L}+\frac{1}{\sqrt{k}}\left(\Lambda+\beta_{i}\right)} e^{2 \pi i h(0)} q^{L^{l}(0)-l / 24} \operatorname{tr}_{M^{\Lambda, \Lambda+\beta_{i}}} O(w) q^{L^{p}(0)-c^{p} / 24}} \sum_{i \in Q / k Q_{L}} \chi_{V_{\sqrt{k} Q_{L}+\frac{1}{\sqrt{k}}\left(\Lambda+\beta_{i}\right)}}(h, q) Z_{M^{\Lambda, \Lambda+\beta_{i}}}(w, q) .
\end{aligned}
$$

Note that $\chi_{V_{\sqrt{k} Q_{L}+\frac{1}{\sqrt{k}}\left(\Lambda+\beta_{i}\right)}}(h, q)$ converges to a holomorphic function $\chi_{V_{\sqrt{k} Q_{L}+\frac{1}{\sqrt{k}}\left(\Lambda+\beta_{i}\right)}}(h, \tau)$ [29] and $Z_{M^{\Lambda, \Lambda+\beta_{i}}}(w, q)$ converges to a holomorphic function $Z_{M^{\Lambda, \Lambda+\beta_{i}}}(w, \tau)$ [36]. Thus $\chi_{L_{\widehat{\mathfrak{g}}}(k, \Lambda)}(w, h, q)$ converges.

For short, we set $\chi_{\Lambda}(w, h, \tau)=\chi_{L_{\widehat{\mathfrak{g}}}(k, \Lambda)}(w, h, \tau)$.

(b) By Theorems 3.3, 4.2 and Lemma 4.3 we have

$$
\chi_{\Lambda}\left(w, \frac{h}{\tau}, \frac{-1}{\tau}\right)=\tau^{\mathrm{wt}[w]} e^{\pi i k\langle h, h\rangle / \tau} \sum_{\Lambda^{\prime} \in P_{+}^{k}} S_{\Lambda, \Lambda^{\prime}} \chi_{\Lambda^{\prime}}(w, h, \tau) .
$$

(c) Let $L$ be a positive definite even lattice of rank $l$ with bilinear form $($,$) . Recall$ from [4] and [25] the lattice vertex operator algebra $V_{L}=M(1) \otimes \mathbb{C}[L]$ and its irreducible modules $V_{L+\lambda_{i}}=M(1) \otimes \mathbb{C}\left[L+\lambda_{i}\right]$ where $L^{\circ}=\cup_{i \in L^{\circ} / L}\left(L+\lambda_{i}\right)$. Then $V_{L}$ is a vertex operator algebra satisfying V1-V3. In this case

$$
\chi_{V_{L+\lambda_{i}}}(h, \tau)=\operatorname{tr}_{V_{L+\lambda_{i}}} e^{2 \pi i h(0)} q^{L(0)-l / 24}=\frac{\theta_{L+\lambda_{i}}(h, \tau)}{\eta(\tau)^{l}}
$$

where

$$
\theta_{L+\lambda_{i}}(h, \tau)=\sum_{\lambda \in L+\lambda_{i}} e^{2 \pi i(h, \lambda)} q^{(\lambda, \lambda) / 2}
$$


It is well known that $\left\{\theta_{L+\lambda} \mid L+\lambda \in L^{\circ} / L\right\}$ are linearly independent functions on $\mathfrak{h} \times \mathbb{H}$ where $\mathfrak{h}=\mathbb{C} \otimes_{\mathbb{Z}} L$. Thus, $\left\{\chi_{L+\lambda} \mid L+\lambda \in L^{\circ} / L\right\}$ are linearly independent functions on $\mathfrak{h} \times \mathbb{H}$. Using the transformation formula

$$
\theta_{L+\lambda}\left(\frac{h}{\tau}, \frac{-1}{\tau}\right)=(-i \tau)^{l / 2}\left|L^{\circ} / L\right|^{-1 / 2} e^{\pi i(h, h) / \tau} \sum_{\lambda^{\prime}+L \in L^{\circ} / L} e^{-2 \pi i\left(\lambda, \lambda^{\prime}\right)} \theta_{L+\lambda^{\prime}}(h, \tau)
$$

and

$$
\eta(-\mathbf{1} / \tau)=(-i \tau)^{1 / 2} \eta(\tau)
$$

we see that

$$
\chi_{L+\lambda_{i}}\left(\frac{h}{\tau}, \frac{-1}{\tau}\right)=\left|L^{\circ} / L\right|^{-1 / 2} e^{\pi i(h, h) / \tau} \sum_{j \in L^{\circ} / L} e^{-2 \pi i\left(\lambda_{i}, \lambda_{j}\right)} \chi_{L+\lambda_{j}}(h, \tau) .
$$

Set $S_{L+\lambda, L+\lambda^{\prime}}=\left|L^{\circ} / L\right|^{-1 / 2} e^{-2 \pi i\left(\lambda, \lambda^{\prime}\right)}$ for $L+\lambda, L+\lambda^{\prime} \in L^{\circ} / L$. Also set

$$
S_{L}=\left(S_{L+\lambda, L+\lambda^{\prime}}\right)_{L+\lambda, L+\lambda^{\prime} \in L^{\circ} / L}
$$

which is the $S$-matrix for the lattice vertex operator algebra $V_{L}$.

(d) Let $L=\sqrt{k} Q_{L}$. Then $L^{\circ}=\frac{1}{\sqrt{k}} P[1$. As in [29] we consider column vector

$$
\overrightarrow{\chi(w, h, \tau)}=\left(\chi_{\Lambda}\right)_{\Lambda \in P_{+}^{k}}, \quad \overrightarrow{\chi_{\sqrt{k} Q_{L}}(h, \tau)}=\left(\chi_{L+\lambda}\right)_{L+\lambda \in L^{\circ} / L}
$$

Let $S_{A}=\left(S_{\Lambda, \Lambda^{\prime}}\right)_{\Lambda, \Lambda^{\prime} \in P_{+}^{k}}$ which is the $S$-matrix for affine vertex operator algebra $L_{\widehat{\mathfrak{g}}}(k, 0)$ (see Lemma 4.3). Also consider the matrix

$$
Z(w, \tau)=\left(Z_{M^{\Lambda, \lambda}}\right)_{\Lambda \in P_{+}^{k}, \lambda+L \in L^{\circ} / L}
$$

where $Z_{M^{\Lambda, \lambda}}=0$ if $\lambda$ does not lie in $\Lambda+Q$. From the discussion above we see that

$$
\overrightarrow{\chi(w, h, \tau)}=Z(w, \tau) \overrightarrow{\chi_{\sqrt{k} Q_{L}}(h, \tau)} .
$$

Performing the transformation of both sides by matrix $\left(\begin{array}{cc}0 & -1 \\ 1 & 0\end{array}\right)$ we see that

$$
\tau^{\mathrm{wt}[w]} e^{\pi i k\langle h, h\rangle / \tau} S_{A} \overrightarrow{\chi(w, h, \tau)}=Z\left(w, \frac{-1}{\tau}\right) e^{\pi i k\langle h, h\rangle / \tau} S_{L} \overrightarrow{\chi_{\sqrt{k} Q_{L}}(h, \tau)} .
$$

Or equivalently,

$$
\tau^{\mathrm{wt}[w]} S_{A} Z(w, \tau) \overrightarrow{\chi_{\sqrt{k} Q_{L}}(h, \tau)}=Z\left(w, \frac{-1}{\tau}\right) S_{L} \overrightarrow{\chi_{\sqrt{k} Q_{L}}(h, \tau)} .
$$

The linear independence of functions $\left\{\chi_{L+\lambda} \mid L+\lambda \in L^{\circ} / L\right\}$ implies that

$$
\tau^{\mathrm{wt}[w]} S_{A} Z(w, \tau)=Z\left(w, \frac{-1}{\tau}\right) S_{L}
$$


It is well known from [29] that $S_{L}$ is symmetric, unitary and $S_{L}^{-1}=\overline{S_{L}}$. In fact, these properties hold for the $S$-matrix associated to any strong rational vertex operator algebra [13]. Finally we deduce

$$
Z\left(w, \frac{-1}{\tau}\right)=\tau^{\mathrm{wt}[w]} S_{A} Z(w, \tau) \overline{S_{L}}
$$

(e) Comparing the $(\Lambda, \lambda)$-entries of the both sides gives

$$
\begin{aligned}
& Z_{M^{\Lambda, \lambda}}\left(w, \frac{-1}{\tau}\right)=\tau^{\mathrm{wt}[w]} \sum_{\Lambda^{\prime} \in P_{+}^{k}, \lambda^{\prime} \in P / k Q_{L}} S_{\Lambda, \Lambda^{\prime}} \overline{S_{\lambda, \lambda^{\prime}}} Z_{M^{\Lambda^{\prime}, \lambda^{\prime}}}(w, \tau) \\
& =\tau^{\mathrm{wt}[w]}\left|P / k Q_{L}\right|^{-1 / 2} \sum_{\Lambda^{\prime} \in P_{+}^{k}, \lambda^{\prime} \in P / k Q_{L}} S_{\Lambda, \Lambda^{\prime}} \epsilon^{2 \pi i \frac{\left\langle\lambda, \lambda^{\prime}\right\rangle}{k}} Z_{M^{\Lambda^{\prime}, \lambda^{\prime}}}(w, \tau) .
\end{aligned}
$$

Now we take $\lambda=\Lambda+\beta_{i}$ for $i \in Q / k Q_{L}$. Note that $M^{\Lambda^{\prime}, \lambda^{\prime}}$ is nonzero if and only if $\lambda^{\prime}+k Q_{L}=\Lambda^{\prime}+\beta_{j}+k Q_{L}$ for some $j \in Q / k Q_{L}$. As a result, we see that

$$
Z_{M^{\Lambda, \Lambda+\beta_{i}}}\left(w, \frac{-1}{\tau}\right)=\tau^{\mathrm{wt}[w]} \sum_{\Lambda^{\prime} \in P_{+}^{k}, j \in Q / k Q_{L}} S_{\left(\Lambda, \Lambda+\beta_{i}\right),\left(\Lambda^{\prime}, \Lambda^{\prime}+\beta_{j}\right)}^{P} Z_{M^{\Lambda^{\prime}, \Lambda^{\prime}+\beta_{j}}}(w, \tau)
$$

and the proof is complete.

\section{ConneCtion With ORBIFOld Theory}

Set $H=\frac{1}{k} P$. For $\alpha \in H$ we define $g_{\alpha}=e^{2 \pi i \alpha(0)}$ where we have identify $\mathfrak{h}$ with $\mathfrak{h}^{*}$ via the bilinear form $\langle$,$\rangle . Then g_{\alpha}$ acts on $L_{\widehat{\mathfrak{g}}}(k, \Lambda)$ for any $\Lambda \in P_{+}^{k}$ such that

$$
g_{\alpha} Y(u, z) g_{\alpha}^{-1}=Y\left(g_{\alpha} u, z\right)
$$

for $u \in L_{\widehat{\mathfrak{g}}}(k, 0)$. In particular, $g_{\alpha}$ is an automorphism of vertex operator algebra $L_{\widehat{\mathfrak{g}}}(k, 0)$. Moreover, $g_{\alpha}=1$ on $L_{\widehat{\mathfrak{g}}}(k, 0)$ if and only if $\alpha \in Q^{\circ}$. That is, $G=H / Q^{\circ}$ is an automorphism group of $L_{\widehat{\mathfrak{g}}}(k, 0)$. For each $\beta \in Q$ we define an irreducible character $\mu_{\beta}$ of $G$ such that $\mu_{\beta}\left(g_{\alpha}\right)=g_{\alpha}(\beta)$. Following [15] and [8] we use $L_{\mathfrak{\mathfrak { g }}}(k, 0)^{\mu_{\beta}}$ to denote the subspace of $L_{\widehat{\mathfrak{g}}}(k, 0)$ which is a sum of irreducible $G$-submodule with character $\mu_{\beta}$. Recall that $Q=\cup_{i \in Q / k Q_{L}}\left(k Q_{L}+\beta_{i}\right)$. By Lemma 5.2, $\left\{\mu_{\beta_{i}} \mid i \in Q / k Q_{L}\right\}$ gives a complete list of inequivalent irreducible characters of $G$. The following result is immediate from [8].

Lemma 7.1. The $L_{\mathfrak{g}}(k, 0)$ is a completely reducible $V_{\sqrt{k} Q_{L}} \otimes K(\mathfrak{g}, k)$-module

$$
L_{\widehat{\mathfrak{g}}}(k, 0)=\bigoplus_{i \in Q / k Q_{L}} L_{\widehat{\mathfrak{g}}}(k, 0)^{\mu_{\beta_{i}}}
$$

and $L_{\widehat{\mathfrak{g}}}(k, 0)^{\mu_{\beta_{i}}}=V_{\sqrt{k} Q_{L}+\frac{1}{\sqrt{k}} \beta_{i}} \otimes M^{0, \beta_{i}}$ is an irreducible module for $V_{\sqrt{k} Q_{L}} \otimes K(\mathfrak{g}, k)$. Moreover, if $i \neq j$ then $L_{\widehat{\mathfrak{g}}}(k, 0)^{\mu_{\beta_{i}}}$ and $L_{\widehat{\mathfrak{g}}}(k, 0)^{\mu_{\beta_{j}}}$ are inequivalent. 
If $\Lambda \in P_{+}^{k}$ is not zero, $L_{\widehat{\mathfrak{g}}}(k, \Lambda)$ is still a module for $H$ but not a module for $G$ unless $\Lambda \in Q$. However, $\alpha \mapsto \tilde{g}_{\alpha}=g_{\alpha} e^{-2 \pi i\langle\alpha, \Lambda\rangle}$ gives a $G$-module structure on $L_{\widehat{\mathfrak{g}}}(k, \Lambda)$. It is clear that

$$
\tilde{g}_{\alpha} Y(u, z) \tilde{g}_{\alpha}^{-1}=Y\left(g_{\alpha} u, z\right)
$$

on $L_{\widehat{\mathfrak{g}}}(k, \Lambda)$. A generalization of Lemma 7.1 is the following:

Lemma 7.2. The $L_{\widehat{\mathfrak{g}}}(k, \Lambda)$ is a completely reducible $V_{\sqrt{k} Q_{L}} \otimes K(\mathfrak{g}, k)$-module

$$
L_{\widehat{\mathfrak{g}}}(k, \Lambda)=\bigoplus_{i \in Q / k Q_{L}} L_{\widehat{\mathfrak{g}}}(k, \Lambda)^{\mu_{\beta_{i}}}
$$

and $L_{\widehat{\mathfrak{g}}}(k, \Lambda)^{\mu_{\beta_{i}}}=V_{\sqrt{k} Q_{L}+\frac{1}{\sqrt{k}}\left(\Lambda+\beta_{i}\right)} \otimes M^{\Lambda, \Lambda+\beta_{i}}$ is an irreducible module for $V_{\sqrt{k} Q_{L}} \otimes K(\mathfrak{g}, k)$. Moreover, if $i \neq j$ then $L_{\widehat{\mathfrak{g}}}(k, \Lambda)^{\mu_{\beta_{i}}}$ and $L_{\widehat{\mathfrak{g}}}(k, \Lambda)^{\mu_{\beta_{j}}}$ are inequivalent.

We can strengthen Lemma 7.2 :

Proposition 7.3. For $\Lambda \in P_{+}^{k}$ and $i \in Q / k Q_{L}, L_{\widehat{\mathfrak{g}}}(k, \Lambda)^{\mu_{\beta_{i}}}$ are inequivalent irreducible $V_{\sqrt{k} Q_{L}} \otimes K(\mathfrak{g}, k)$-modules.

Proof. From equation (5.1) we know that $L_{\widehat{\mathfrak{g}}}(k, \Lambda) \circ g_{\alpha}$ and $L_{\widehat{\mathfrak{g}}}(k, \Lambda)$ are isomorphic $L_{\widehat{\mathfrak{g}}}(k, 0)$-modules where $L_{\widehat{\mathfrak{g}}}(k, \Lambda) \circ g_{\alpha}=L_{\widehat{\mathfrak{g}}}(k, \Lambda)$ as vector spaces and $Y_{L_{\widehat{\mathfrak{g}}}(k, \Lambda) \circ g_{\alpha}}(v, z)=$ $Y_{L_{\mathfrak{\mathfrak { g }}}(k, \Lambda)}\left(g_{\alpha} v, z\right)$ for $v \in L_{\widehat{\mathfrak{g}}}(k, 0)$. According to a general result in orbifold theory [23], [18], $L_{\widehat{\mathfrak{g}}}(k, \Lambda)^{\mu_{\beta_{i}}}$ are inequivalent irreducible $V_{\sqrt{k} Q_{L}} \otimes K(\mathfrak{g}, k)$-modules.

We can now express the trace functions $Z_{M^{\Lambda . \Lambda+\beta_{i}}}(w, \tau)$ in terms of $\chi_{\Lambda}(w, \alpha, \tau)$ and the characters of irreducible modules for lattice vertex operator algebra $V_{L}$ with $L=\sqrt{k} Q_{L}$. For $w \in K(\mathfrak{g}, k)$ we have

$$
\begin{aligned}
& Z_{V_{\sqrt{k} Q_{L}+\frac{1}{\sqrt{k}}\left(\Lambda+\beta_{i}\right)} \otimes M^{\Lambda, \Lambda+\beta_{i}}}(w, \tau)=\frac{1}{\left|Q / k Q_{L}\right|} \sum_{\alpha \in G} \operatorname{tr}_{L_{\widehat{\mathfrak{g}}}(k, \Lambda)} O(w) \tilde{g}_{\alpha} q^{L(0)-c / 24} e^{-2 \pi i\left\langle\alpha, \beta_{i}\right\rangle} \\
& =\frac{1}{\left|Q / k Q_{L}\right|} \sum_{\alpha \in G} \operatorname{tr}_{L_{\widehat{\mathfrak{g}}}(k, \Lambda)} O(w) e^{2 \pi i \alpha(0)} q^{L(0)-c / 24} e^{-2 \pi i\left\langle\alpha, \beta_{i}+\Lambda\right\rangle} \\
& =\frac{1}{\left|Q / k Q_{L}\right|} \sum_{\alpha \in G} e^{-2 \pi i\left\langle\alpha, \beta_{i}+\Lambda\right\rangle} \chi_{\Lambda}(w, \alpha, \tau) .
\end{aligned}
$$

This implies the following:

Proposition 7.4. For $\Lambda \in P_{+}^{k}, i \in Q / k Q_{L}$ and $w \in K(\mathfrak{g}, k)$ we have

$$
Z_{M^{\Lambda, \Lambda+\beta_{i}}}(w, \tau)=\frac{1}{\left|Q / k Q_{L}\right|} \frac{\eta(\tau)^{l}}{\theta_{\sqrt{k} Q_{L}+\frac{1}{\sqrt{k}}\left(\Lambda+\beta_{i}\right)}(\tau)} \sum_{\alpha \in G} e^{-2 \pi i\left\langle\alpha, \beta_{i}+\Lambda\right\rangle} \chi_{\Lambda}(w, \alpha, \tau) .
$$




\section{REFERENCES}

[1] C. Ai, C. Dong, X. Jiao and L. Ren, The irreducible modules and fusion rules for the Parafermion vertex operator algebras, Trans. AMS.,370 (2018), 5963-5981.

[2] T. Arakawa, C.H. Lam and H. Yamada, Zhu's algebra, $C_{2}$-algebra and $C_{2}$-cofiniteness of parafermion vertex operator algebras, Adv. Math. 264 (2014), 261-295.

[3] T. Arakawa, C.H. Lam and H. Yamada, Parafermion vertex operator algebras and W-algebras, DOI: https://doi-org.oca.ucsc.edu/10.1090/tran/7547

[4] R. E. Borcherds, Vertex algebras, Kac-Moody algebras, and the Monster, Proc. Natl. Acad. Sci. USA 83 (1986), 3068-3071.

[5] S. Carnahan and M. Miyamoto, Regularity of fixed-point vertex operator subalgebras, arXiv:1603.05645,

[6] C. Dong, C.H. Lam and H. Yamada, W-algebras related to parafermion algebras, J. Algebra 322 (2009), 2366-2403.

[7] C. Dong, C.H. Lam, Q. Wang and H. Yamada, The structure of parafermion vertex operator algebras, J. Algebra 323 (2010), 371-381.

[8] C. Dong, H. Li and G. Mason, Compact automorphism groups of vertex operator algebras, International Math. Research Notices 18 (1996), 913-921.

[9] C. Dong, H. Li and G. Mason, Regularity of rational vertex operator algebras, Adv. Math. 132 (1997), 148-166.

[10] C. Dong, H. Li and G. Mason, Twisted representations of vertex operator algebras, Math. Ann. 310 (1998), 571-600.

[11] C. Dong, H. Li and G. Mason, Modular invariance of trace functions in orbifold theory and generalized moonshine, Comm. Math. Phys. 214 (2000), 1-56.

[12] C. Dong, H. Li, G. Mason and P. Montague, The radical of a vertex operator algebra, in: Proc. of the Conference on the Monster and Lie algebras at The Ohio State University, May 1996, ed. by J. Ferrar and K. Harada, Walter de Gruyter, Berlin-New York, 1998, 17-25.

[13] C. Dong, X. Lin and S.-H. Ng, Congruence property in conformal field theory, Algebra 8 Number Theory 9 (2015), 2121-2166.

[14] C. Dong, K. Liu and X. Ma, Elliptic genus and vertex operator algebras, Quarterly J. Pure and Applied Math. 1 (2005), 791-815.

[15] C. Dong and G. Mason, On quantum Galois theory, Duke Math. J. 86 (1997), 305-321.

[16] C. Dong and G. Mason, Rational vertex operator algebras and the effective central charge, International Math. Research Notices 56 (2004), 2989-3008.

[17] C. Dong and L. Ren, Representations of the parafermion vertex operator algebras, Adv. Math. 315 (2017), 88-101.

[18] C. Dong, L. Ren and F. Xu, On orbifold theory, Adv. Math. 321 (2017), 1-30.

[19] C. Dong and Q. Wang, The structure of parafermion vertex operator algebras: general case, Comm. Math. Phys. 299 (2010), 783-792.

[20] C. Dong and Q. Wang, On $C_{2}$-cofiniteness of the parafermion vertex operator algebras, $J$. Algebra 328 (2011), 420-431.

[21] C. Dong and Q. Wang, Parafermion vertex operator algebras, Frontiers of Mathematics in China 6(4) (2011), 567-579. 
[22] C. Dong and Q. Wang, Quantum dimensions and fusion rules for parafermion vertex operator algebras, Proc. AMS 144 (2016), 1483-1492.

[23] C. Dong and G. Yamskulna, Vertex operator algebras, Generalized double and dual pairs, Math. Z. 241 (2002), 397-423.

[24] I. Frenkel, Y. Huang and J. Lepowsky, On axiomatic approaches to vertx operator algebras and modules, Mem. AMS 104, 1993.

[25] I. B. Frenkel, J. Lepowsky and A. Meurman, Vertex Operator Algebras and the Monster, Pure and Applied Math., Vol. 134, Academic Press, Boston, 1988.

[26] I. B. Frenkel and Y. Zhu, Vertex operator algebras associated to representations of affine and Virasoro algebras, Duke Math. J. 66 (1992), 123-168.

[27] J. Humphreys, Introduction to Lie algebras and representation theory, Graduate Texts in Mathematics, 9. Springer-Verlag, New York-Berlin, 1978.

[28] C. Jiang and Q. Wang, Representations of Z2-orbifold of the parafermion vertex operator algebra $K\left(s l_{2}, k\right)$, arXiv:1712.07277.

[29] V. G. Kac, Infinite-dimensional Lie Algebras, 3rd ed., Cambridge University Press, Cambridge, 1990.

[30] V. Kac and D. Peterson, Infinite-dimensional Lie algebras, theta functions and modular forms, Adv. in Math. 53 (1984), 125-264.

[31] M. Krauel, One-point theta functions for vertex operator algebras, J. Alg. 481 (2017), 250-272.

[32] J. Lepowsky and H. Li, Introduction to Vertex Operator Algebras and Their Representations, Progress in Math., Vol. 227, Birkhäuser, Boston, 2004.

[33] H. Li, The physics superselection principle in vertex operator algebra theory, J. Alg. 196 (1997), 436-457.

[34] H. Li, Certain extensions of vertex operator algebras of affine type, Comm. Math. Phys. 217 (2001), 653-696.

[35] M. Miyamoto, A modular invariance of the theta functions defined on vertex operator algebras, Duke Math. 101 (2000), 221-236.

[36] Y. Zhu, Modular invariance of characters of vertex operator algebras, J. Amer, Math. Soc. 9 (1996), 237-302.

Department of Mathematics, University of California, Santa Cruz, CA 95064

E-mail address: dong@ucsc.edu

Department of Mathematics, Mit, 77 Mass. Ave, Cambridge, Ma 02139

E-mail address: kac@math.mit.edu

School of Mathematics, Sichuan University, Chengdu 610064 China

E-mail address: renl@scu.edu.cn 